\title{
University of Glasgow
}

Zhang, R., Yang, K., Abbasi, Q. H., Qaraqe, K. A. and Alomainy, A. (2018)

Analytical modelling of the effect of noise on the terahertz in-vivo

communication channel for body-centric nano-networks. Nano

Communication Networks, 15, pp. 59-68.

(doi:10.1016/j.nancom.2017.04.001)

This is the author's final accepted version.

There may be differences between this version and the published version. You are advised to consult the publisher's version if you wish to cite from it.

http://eprints.gla.ac.uk/140612/

Deposited on: 04 May 2017

Enlighten - Research publications by members of the University of Glasgow http://eprints.gla.ac.uk 


\title{
Analytical Modelling of the Effect of Noise on the Terahertz In-vivo Communication Channel for Body-centric Nano-networks
}

\author{
Rui Zhang, ${ }^{\mathrm{a}, *}$ Ke Yang $^{\mathrm{a}}$, Qammer H. Abbasi ${ }^{\mathrm{a}, \mathrm{b}, \mathrm{c}}$, Khalid A. Qaraqe ${ }^{\mathrm{c}}$, Akram Alomainy ${ }^{\mathrm{a}}$ \\ ${ }^{a}$ Department of Electronic Engineering and Computer Science, Queen Mary University of London, UK \\ ${ }^{b}$ School of Engineering, University of Glasgow, UK \\ ${ }^{c}$ Department of Electrical and Computer Engineering, Texas A\&M University at Qatar, Doha, Qatar
}

\begin{abstract}
The paper presents an analytical model of the terahertz $(\mathrm{THz})$ communication channel $(0.1$ - $10 \mathrm{THz}$ ) for in-vivo nano-networks by considering the effect of noise on link quality and information rate. The molecular absorption noise model for in-vivo nano-networks is developed based on the physical mechanisms of the noise present in the medium, which takes into account both the radiation of the medium and the molecular absorption from the transmitted signal. The signal-to-noise ratio (SNR) of the communication channel is investigated for different power allocation schemes and the maximum achievable information rate is studied to explore the potential of $\mathrm{THz}$ communication inside the human body. The obtained results show that the information rate is inversely proportional to the transmission distance. Based on the studies on channel performance, it can be concluded that the achievable transmission distance of in-vivo $\mathrm{THz}$ nano-networks should be restrained to approximately $2 \mathrm{~mm}$ maximum, while the operation band of in-vivo $\mathrm{THz}$ nano-networks should be limited to the lower band of the $\mathrm{THz}$ band. This motivates the utilisation of hierarchical/cooperative networking concepts and hybrid communication techniques using molecular and electromagnetic methods for future body-centric nano-networks.
\end{abstract}

Keywords: Body-centric communication, Nano-networks, Noise modelling, $\mathrm{THz}$ channel modelling

\section{Introduction}

Nanotechnology is an emerging field of research with the potential to introduce entirely new scientific innovations. Nanotechnology enables the miniaturization and fabrication of devices in a scale ranging from one to a few hundred nanometres. The interconnection of nano-devices in a network or nano-network will expand the capabilities and applications of

\footnotetext{
*Corresponding author

Email addresses: rui.zhang@qmul.ac.uk (Rui Zhang), k.yang@qmul.ac.uk (Ke Yang), Qammer.Abbasi@glasgow.ac.uk (Qammer H. Abbasi), k.qaraqe@qatar.tamu.edu (Khalid A. Qaraqe), a.alomainy@qmul.ac.uk (Akram Alomainy)
} 
individual nano-devices [1]. Due to the advantages of small size, bio-compatibility and biostability, this small-scale network is especially promising in biomedical fields, such as medical diagnostics and selective sickness treatment $[2,3]$. Two main alternatives for communication at nanoscale, namely molecular communication and electromagnetic (EM) communication are proposed and widely explored. Intra-body wireless communication at the optical frequencies has been studied in [4] showing that the propagation of EM waves at optical frequencies inside the human body is mainly affected by the scattering from cells. The latest advancements in graphene-based electronics have opened the door to EM communication among nano-devices in the $\mathrm{THz}$ band inside human tissues [2]. Besides, the envisioned EM nano-communication is handled in the $\mathrm{THz}$ band because of its non-ionisation hazards for biological tissues and less susceptibility to some of the propagation effects (i.e., Rayleigh scattering etc.) [5,6]. Some studies have been conducted on the applicability of $\mathrm{THz}$ communication for biomedical applications in $[2,7,8]$, which motivates further investigation on the channel characterisation of the in-vivo nano-networks at the $\mathrm{THz}$ band and the development of novel models for system performance assessment and link quality analysis.

To date, a limited number of studies have been performed on the channel characterisation and model of nano-communication at the $\mathrm{THz}$ band. Channel modelling of the $\mathrm{THz}$ wave propagating in the atmosphere with a different concentration of water vapour and the corresponding channel capacity for different power allocation schemes were studied in $[9,10]$. Subsequently, both numerical and analytical EM channel modelling at the $\mathrm{THz}$ band for in-body nano-communication were investigated in [11]. In order to quantify the potential of the $\mathrm{THz}$ communication, and to proceed with realistic and accurate representation of the actual capacity and channel model in the $\mathrm{THz}$ band, consideration of channel noise model is necessary.

In the open literature, some studies have been performed on the $\mathrm{THz}$ channel noise characterisation $[9,10,11]$. A noise model for nano-communication channels in the atmosphere was first introduced in [9], approximating the noise temperature as the molecular absorption noise, which is considered as a basis in $[12,13]$. The same noise model was applied in $[11,14]$ to characterise the in-vivo nano-communication channel. However, this noise model only takes into account the radiation energy from the medium and focuses on the transformation of the energy directly into antenna temperature, while ignoring the molecular absorption from the transmitted signal. The noise model was updated in [15] where the statement was proposed that the molecular absorption noise is composed of the atmospheric noise and the self-induced noise, and the molecular absorption of the transmitted signal should be an additional noise source in the $\mathrm{THz}$ band. Such models were further investigated and applied in $[16,17,18]$ to evaluate the channel performance, such as mutual information rate and bit error rate. However, the atmospheric noise is only valid for $\mathrm{THz}$ communication in the atmosphere. Furthermore, a general background noise is provided based on the physical causes and mechanisms behind the molecular absorption noise in [19], which pave the way for the investigation on the noise model for the in-vivo scenario in this paper. Nevertheless, the molecular absorption noise model developed in [19] is not fully comprehensive, since the distance-dependent spreading path loss is not considered, which should also be added in the energy conservation to provide a clearer and more complete 
understanding of the noise influence on the channel and network quality. The noise model of in-vivo communication is studied based on the physical mechanism of the channel noise in the medium, which takes into account both the radiation of the medium and the molecular absorption from the transmitted signal in [20].

In this paper, the noise model for in-vivo nano-communication is developed, which is considered as the combined results of the background noise and the self-induced noise. The updated noise model is applied to calculate the Signal-to-Noise Ratio (SNR) of the communication channel in order to evaluate the link quality. Furthermore, to quantify the potential of the $\mathrm{THz}$ band for communication in the human body, the information rate with a single user case has been studied.

The remainder of the paper is organised as follows. In section 2, the basics of molecular absorption and path loss model for EM communication at the $\mathrm{THz}$ band is provided. The physical mechanisms of the molecular absorption noise, the noise model for the in-vivo nanonetworks with the consideration of the physical background and derivation of the model is developed and the molecular absorption noise power spectral density (p.s.d) for different human tissues is discussed and analysed in section 3. SNR of the nano-communication inside human tissues for different power allocation schemes is studied in section 4 . The information rate of the in-vivo nano-networks at the $\mathrm{THz}$ band is investigated in section 5 . Finally, the paper is concluded in section 6 .

\section{Theoretical and analytical considerations}

In this section, the analysis of the end-to-end (including channel) noise model including transmission, propagation and reception of the EM wave is performed. Considering the complexity of the real human tissues, two assumptions are made here:

- A spherically symmetric propagation environment is assumed with the receiver at the centre of the sphere and the transmitter at the distance $r$ from the receiver.

- The antennas of the transmitter are assumed to be ideal isotropic ones.

\subsection{Molecular absorption}

Molecular absorption is a process that the EM energy is partially transformed to kinetic energy internal to vibrating molecules [10], which can be described by the absorption coefficient. Because the vibration frequencies at which a given molecule resonates change with the internal structure of the molecule [10], this quantity depends on the frequency and gives the $\mathrm{THz}$ band a unique frequency-selective spectral absorption profile. Given the absorption coefficient, the amount of incident EM radiation that is capable of propagating through the absorbing medium at a given frequency can be calculated. This parameter is defined by transmittance, which is obtained by using the Beer-Lamberts law as [21, 22],

$$
\tau(r, f)=e^{-\alpha(f) r}
$$

where $f$ is the frequency of the EM wave, $r$ stands for the total path length and $\alpha(f)$ is the absorption coefficient. 


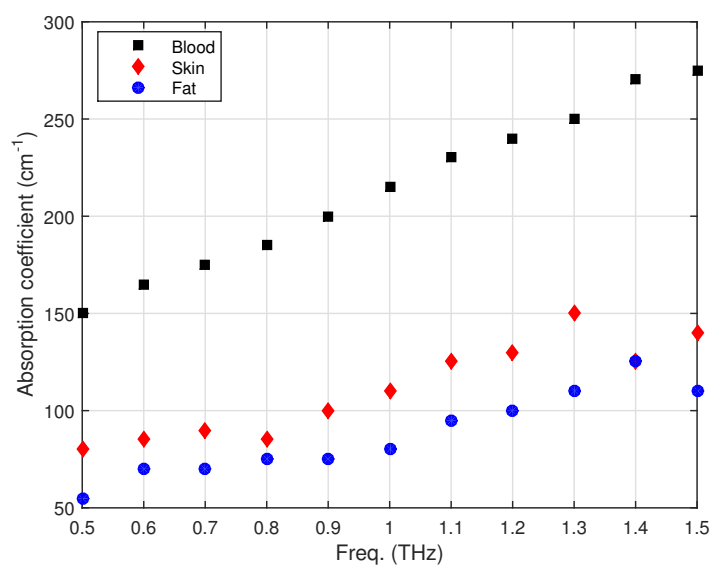

Figure 1: Molecular absorption coefficient as a function of frequency of human blood, skin and fat

Molecular absorption causes attenuation to signals, which can be obtained from the transmittance of the medium $\tau$ given by (1), when travelling a distance $r$ as $[9,10]$,

$$
A_{a b s}(r, f)=\frac{1}{\tau(r, f)}=e^{\alpha(f) r}
$$

In this paper body-centric nano-networks are focused on human blood, skin and fat tissues and their absorption coefficient at the frequency band of interest are shown in Fig. 1. The details on the calculation of the absorption coefficient for human blood, skin and fat tissues can be found in $[11,14]$. Compared to the absorption coefficient in the atmosphere provided in [10], on the one hand, the absorption coefficient in human tissues can be thousands times of that in air at the same frequency. On the other hand, different from the thousand resonant peaks of water vapour over the $\mathrm{THz}$ band, the absorption coefficient of human tissues increases with frequency much more steadily. It gives in-vivo $\mathrm{THz}$ communication some peculiar behaviours, which will be presented in the following sections.

\subsection{Path loss}

The path loss in human tissues is divided into two parts: the spreading loss and molecular absorption loss [9]. The spreading loss is a part of attenuation, caused by the expansion of a wave propagating through the medium and it can be calculated from the modified Friis transmission equation [22],

$$
A_{s p r}(r, f)=\left(\frac{4 \pi n f r}{c}\right)^{2}
$$

where $f$ is the frequency of the EM wave and $n$ is the corresponding refractive index of the $\mathrm{THz}$ wave in the human tissue medium. $4 r^{2}$ denotes the isotropic expansion term and $4 \pi(n f / c)^{2}$ stands for the frequency dependent receiver antenna aperture term. Considering the attenuation, the total path loss can be described as,

$$
A_{P L}(r, f)=A_{s p r}(r, f) A_{a b s}(r, f)=\left(\frac{4 \pi n f r}{c}\right)^{2} e^{\alpha(f) r}
$$


and the expected received signal power can be represented by [23],

$$
P_{R}(r)=\int_{B} S(f)\left(\frac{c}{4 \pi n f r}\right)^{2} e^{-\alpha(f) r} d f
$$

where $S(f)$ is the transmitted signal p.s.d from the transmitter antenna and $B$ is the channel bandwidth.

The dependency of the channel path loss on the distance and the frequency has also been illustrated in [11]. Due to the significantly high absorption coefficient in human tissues, the path loss suffers more from the molecular absorption loss than the spreading loss. More specifically, at the same frequency and transmission distance, the exponential loss caused by the molecular absorption is approximately double the spreading loss contributions to the path loss. Furthermore, it is demonstrated that the path loss increases with both the transmission distance and frequency and that the path loss would reach $80 \mathrm{~dB}$ when the transmission distance increases to $2 \mathrm{~mm}$ for all the three different human tissues [11].

\section{Molecular absorption noise}

\subsection{Noise model}

The noise in the $\mathrm{THz}$ band is primarily contributed by the molecular absorption noise. This kind of noise is caused by vibrating molecules which partially reradiate the energy that has been previously absorbed [10]. Thus, this noise is dependent on the transmitted signal. The total molecular absorption noise p.s.d. $S_{N}$ is proposed to be contributed by the atmospheric noise $S_{N 0}$ and the self-induced noise $S_{N 1}$ in [15], and can be obtained as,

$$
\begin{gathered}
S_{N}(r, f)=S_{N 0}(r, f)+S_{N 1}(r, f) \\
S_{N 0}(f)=\lim _{r \rightarrow \infty} k_{B} T_{0}\left(1-e^{-\alpha(f) r}\right)\left(\frac{c}{\sqrt{4 \pi} f_{0}}\right)^{2} \\
S_{N 1}(r, f)=S(f)\left(1-e^{-\alpha(f) r}\right)\left(\frac{c}{4 \pi f r}\right)^{2}
\end{gathered}
$$

where $r$ refers to the propagation distance, $f$ stands for the frequency of the EM wave, $k_{B}$ is the Boltzmann constant, $T_{0}$ is the reference temperature of the medium, $\alpha(f)$ is the absorption coefficient, $c$ is the speed of light in vacuum, $f_{0}$ is the design centre frequency, and $S$ is the p.s.d of the transmitted signal.

The atmospheric noise is caused by the temperature of the absorbing atmosphere, making the atmosphere (or any medium) an effective black body radiator in homogeneously absorbing medium (in the frequency domain) [19]. This atmospheric noise is therefore known as a background noise, which is independent of the transmitted signal. However, the noise model in (7) is only a special case for $\mathrm{THz}$ communication in air. Without loss of generality, the term $k_{B} T_{0}$ should be replaced with the Plancks law, since it is a general radiative function of the surface of the black body [19]. Consequently, it is believed that the molecular absorption noise is contributed by the background noise and the self-induced noise. 
The background noise caused by the radiation of the medium can be described by the Planck's function [24],

$$
B\left(T_{0}, f\right)=\frac{2 h \pi f^{3}}{c^{2}}\left(e^{\frac{h f}{k_{B} T_{0}}}-1\right)^{-1}
$$

where $k_{B}$ is the Boltzmann's constant and $h$ is Planck constant. Planck's function is multiplied with $\pi$ to transform the unit from $W / H z / \mathrm{cm}^{2} / \mathrm{sr}$ to $W / \mathrm{Hz} / \mathrm{cm}^{2}$.

For simplicity, the transmission medium is assumed to be an isothermal and a homogeneous layer with the thickness $r$. As mentioned above, this background noise is generated by the radiation of the local sources of the medium and it is assumed that this radiation is only from the original energy state of the molecules before transmission happens; thus it is independent of the transmitted signal. The background noise can be described as [24],

$$
\begin{aligned}
& S_{N b}(f)=\int_{0}^{r} B\left(T_{0}, f\right) \alpha(f) e^{-\alpha(f) s} d s \\
& =B\left(T_{0}, f\right)\left(1-e^{-\alpha(f) r}\right) \simeq B\left(T_{0}, f\right)
\end{aligned}
$$

The integral in (10) describes the noise intensity at the centre of a sphere with a radius $r$, given all the points $s$ in the medium contribute to the noise intensity. Since this is obtained by the Planck's function, the unit of the background noise p.s.d is $W / \mathrm{Hz} / \mathrm{cm}^{2}$. The background noise can be further approximated by taking into account the (ideal) antenna aperture term $c^{2} /\left(4 \pi f_{0}\right)^{2}$ to get the background noise p.s.d with the unit $W / H z[19]$,

$$
S_{N b}(f)=B\left(T_{0}, f\right) \frac{c^{2}}{4 \pi f_{0}^{2}}
$$

In terms of the induction mechanism of the self-induced noise, the internal vibration of the molecules turns into the emission of EM radiation at the same frequency of the incident waves that provoked this motion $[9,10]$. It is obtained with the assumption that all the absorbed energy from the transmitted signal received at the receiver would turn into molecular absorption noise as shown in (8), where $(4 \pi r f / c)^{2}$ accounts for the spreading loss.

In addition to the molecular absorption noise, there are other noise sources that can affect the communication performance, such as the device noise. A number of prototypes of antennas emitting at the $\mathrm{THz}$ band available today are built with conventional materials. In this case, the Johnson-Nyquist thermal noise should be taken into account as a source of noise [13]. However, with the development of new materials, it is possible that the thermal noise can be neglected with the use of graphene and its derivatives to create $\mathrm{THz}$ antennas. Graphene-based nanostructures allow the ballistic transport of electrons, leading to very low thermal noise in the device; thus, it is reasonably expected that the noise is minor [25, 26]. Therefore, the molecular absorption noise in the transmission is the dominant contributor to noise at the receiver.

\subsection{In-vivo scenario}

With regards to the in-vivo scenario, the speed of light in the human body could change with the composition of the medium and the frequency of the $\mathrm{THz}$ wave. Therefore, c is 
replaced with $c / n$ in (7-9) and (11), which results in that the total molecular absorption noise p.s.d. $S_{N}$ is contributed by the background noise $S_{N b}$ and the self-induced noise $S_{N s}$, and can be represented as,

$$
\begin{gathered}
S_{N}(r, f)=S_{N b}(r)+S_{N s}(r, f) \\
S_{N b}(f)=B\left(T_{0}, f\right)\left(\frac{c}{\sqrt{4 \pi} n_{0} f_{0}}\right)^{2} \\
S_{N s}(r, f)=S(f)\left(1-e^{-\alpha(f) r}\right)\left(\frac{c}{4 \pi n r f}\right)^{2}
\end{gathered}
$$

where $n$ is the corresponding refractive index of the $\mathrm{THz}$ wave in the medium, when the frequency is $f, f_{0}$ is the design centre frequency and $n_{0}$ is the corresponding refractive index.

It is clearly shown that the background noise depends on the temperature and composition of the medium in (14). It is assumed that the human tissues are isothermal; thus, the background noise changes slightly with the refractive index in different transmission mediums. Moreover, the self-induced noise is dependent on the transmitted signal and for simplicity in this section only transmitted signal with flat p.s.d over the entire frequency is considered to comparatively illustrate the difference between these two kinds of noise. To keep the numerical results realistic, and in light of the state of the art in nano-transceivers, the flat power is adopted with the total energy equal to $1 \mathrm{pJ}$ and the pulse duration be 100 $f s[27,28]$. The background noise and self-induced noise p.s.d for human blood, skin and fat tissues are shown in Fig. 2 and Fig. 3, respectively.

From Fig. 2, it can be clearly seen that the background noise p.s.d is almost the same in different kinds of tissue, because the slight difference of refractive index does not play a significant role in (14). Besides, when sharing the same transmitted signal power, the self-induced noise slightly increases from blood to fat, because the absorption coefficient and refractive index increase with the water concentration in the medium and comparatively blood has higher water proportion than skin and fat. More importantly, it can be seen from Fig. 3 that the noise p.s.d has a steady change with frequency, which is different from the abrupt fluctuation of $\mathrm{THz}$ communication in air as shown in [10]. The reason is that the molecular absorption coefficient has steady increase over the frequency of interest. Furthermore, the self-induced noise p.s.d decreases with distance. Because the self-induced noise is directly proportional to the transmitted signal, and the signal is inversely proportional to the transmission distance. Thus, the self-induced noise is inversely proportional to the distance.

Moreover, comparing Fig. 2 with Fig. 3, the self-induced noise p.s.d is about seven orders of magnitude higher than the background noise in all these different human tissues. The main reason is that the transmitted pulse energy is chosen to be high enough in order for better information transmission. In this case, it can be concluded that the self-induced noise is the dominant noise source and the background noise is negligible in the $\mathrm{THz}$ band for in-vivo nano-networks. 


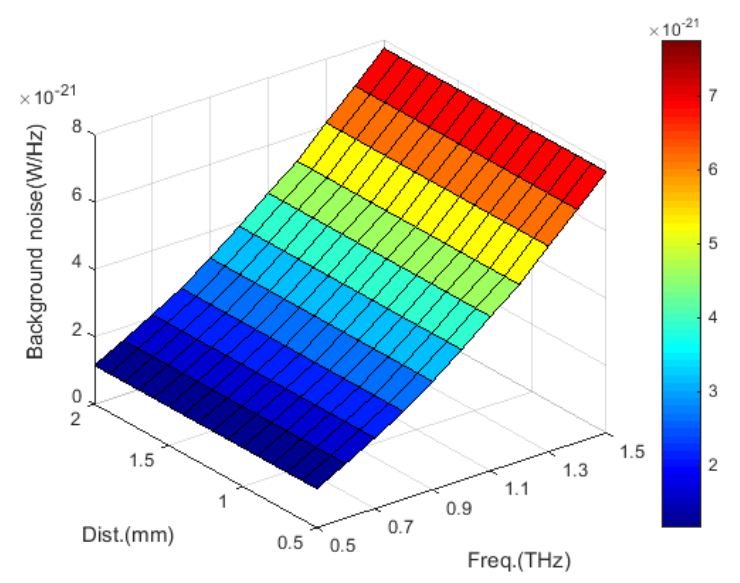

(a) Blood

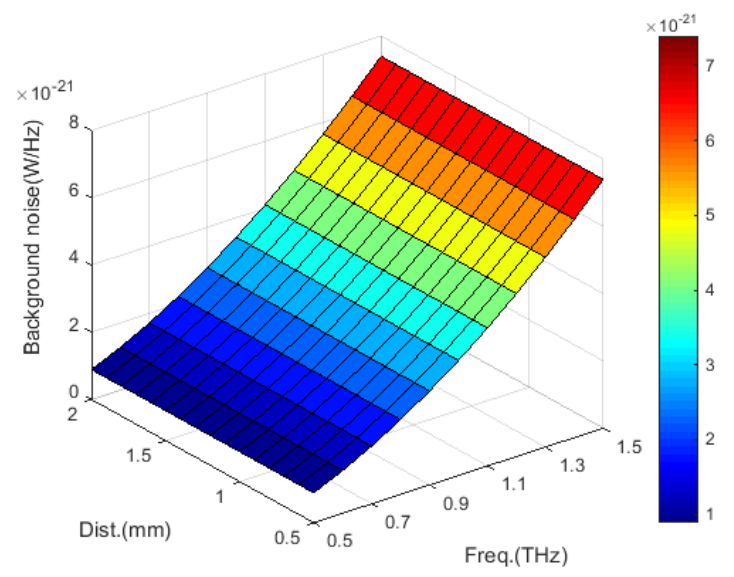

(b) Skin

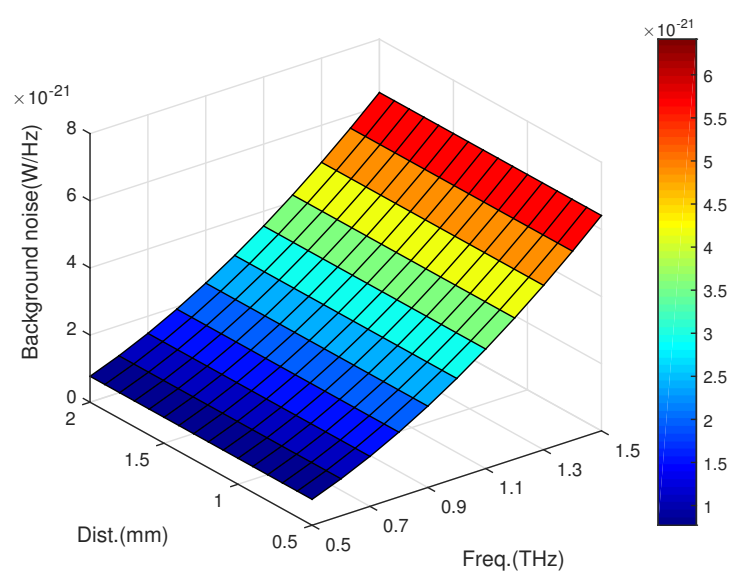

(c) Fat

Figure 2: Background noise p.s.d at terahertz frequencies for different human tissues (a) Blood, (b) Skin and (c) Fat 


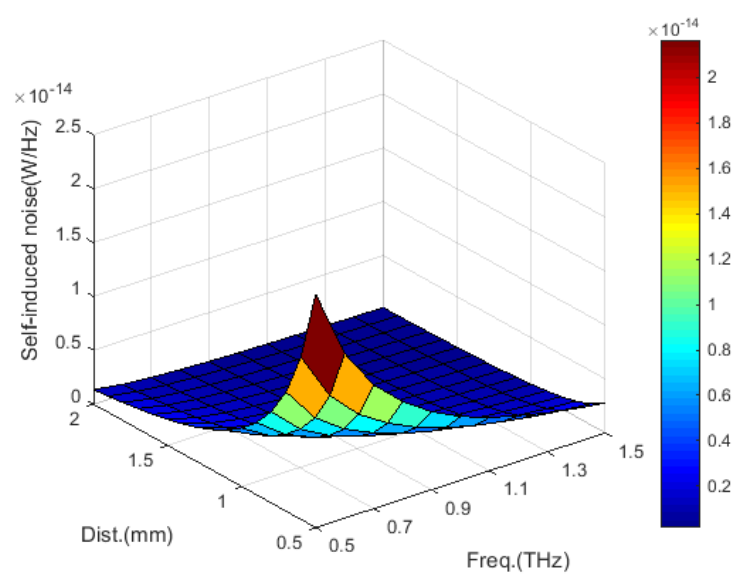

(a) Blood

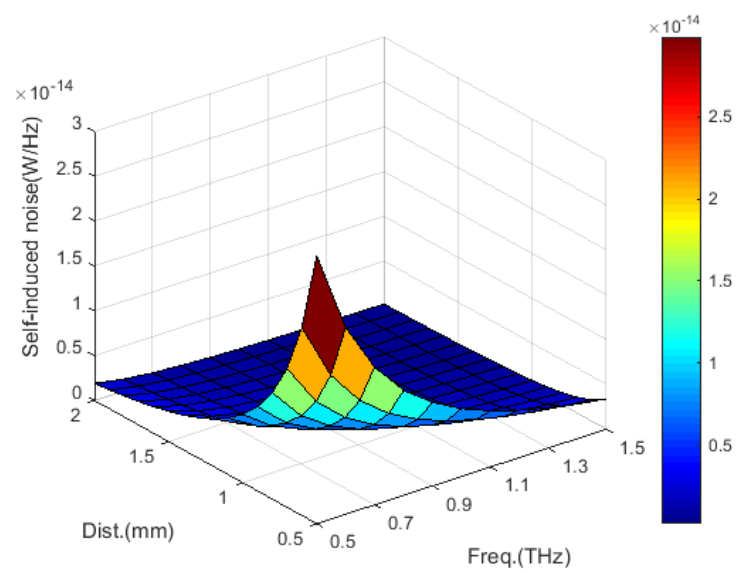

(b) Skin

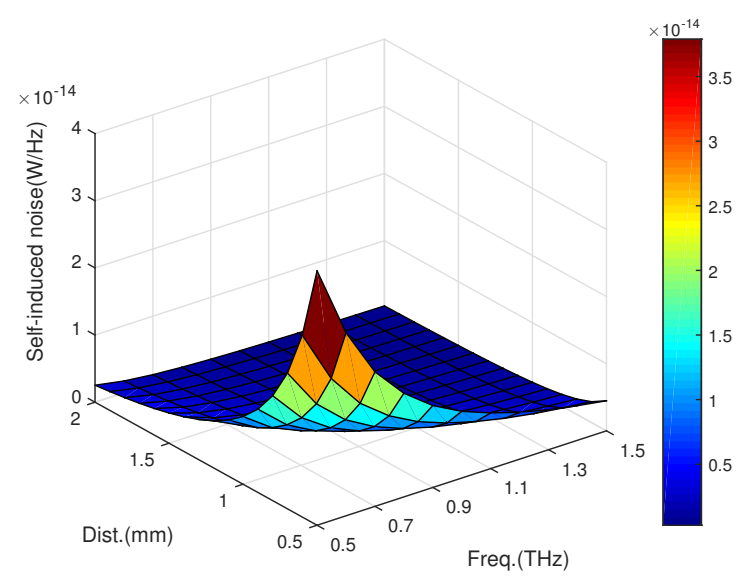

(c) Fat

Figure 3: Self-Induced noise p.s.d at terahertz frequencies for different human tissues (a) Blood, (b) Skin and (c) Fat 


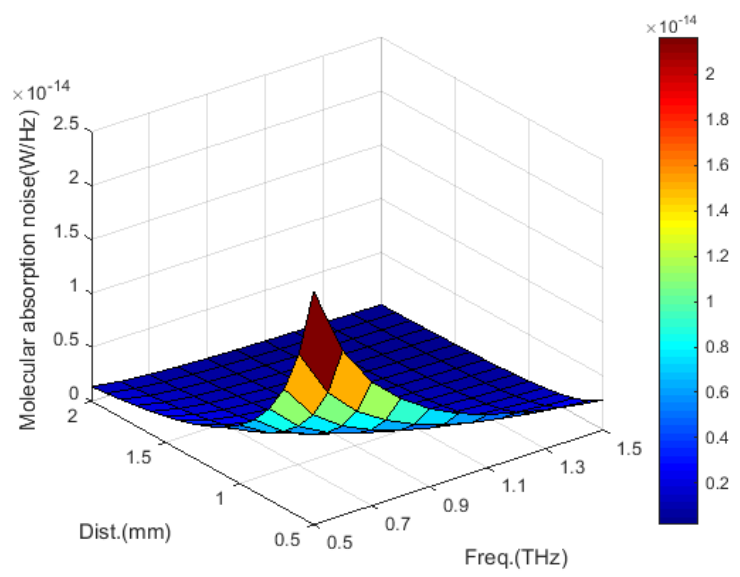

(a) Blood

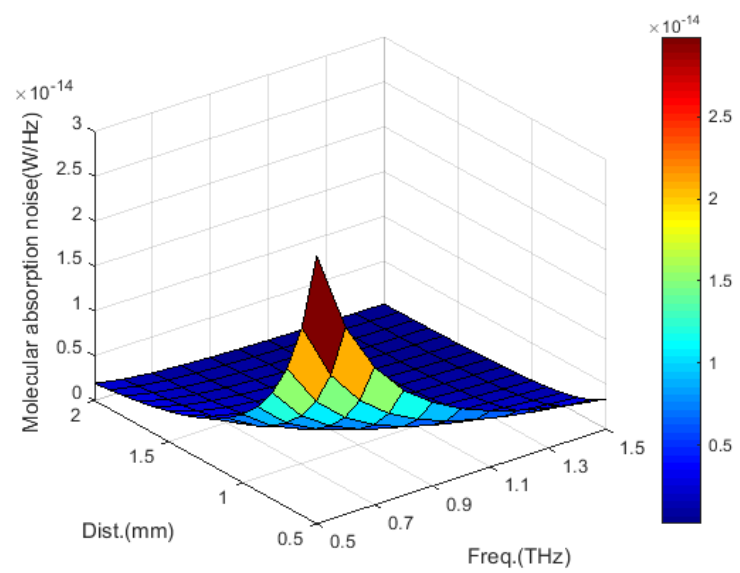

(b) Skin

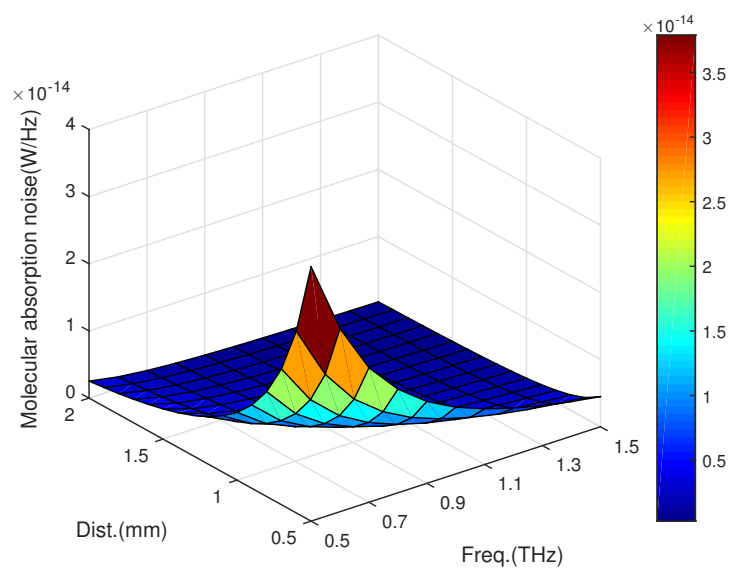

(c) Fat

Figure 4: Molecular absorption noise p.s.d at terahertz frequencies for different human tissues (a) Blood, (b) Skin and (c) Fat 
The molecular absorption noise p.s.d for these considered tissues is illustrated in Fig. 4, which is almost the same with the self-induced noise p.s.d. This highlights the fact that the background noise can be discarded in our analysis.

\section{Power allocation and Signal-to-noise ratio}

For the general communication systems, besides the effect of both path loss and noise, communication capabilities are also strictly influenced by the distribution of power transmission $P_{T}$ in the frequency domain [29]. As discussed in section 3, it is clear that the molecular absorption noise is directly related to the transmitted signal, and when the transmitted signal is high enough, the self-induced noise could be the only noise source in the channel; thus, in this section, two communication schemes (namely, flat and pulse-based) are considered to investigate the SNR of the in-vivo communication at the $\mathrm{THz}$ band.

\subsection{Flat communication}

In the simplest case, the total transmitted signal power $P_{T}$ is uniformly distributed over the entire operative band $(0.5-1.5 \mathrm{THz})$. Thus the corresponding transmitted signal p.s.d is,

$$
S_{\text {flat }}(f)=P_{T} / \text { Bfor } f \in B, \text { otherwise }
$$

\subsection{Pulse-based communication}

The transmitted signal can be modelled with an nth derivative of a Gaussian-shape: $\phi(f)=(2 \pi f)^{2} n e^{(-2 \pi \sigma f)^{2}}[5]$. Thus, the signal p.s.d can be expressed as [10],

$$
S_{p}^{(n)}(f)=a_{0}^{2} \phi(f)
$$

where $\sigma$ and $a_{0}^{2}$ are the standard deviation of Gaussian pulse and a normalising constant, respectively. Considering that $\int_{f_{m}}^{f_{M}} S_{P}^{(n)}(f) d f=P_{T}$, the normalising constant is obtained as $[5]$,

$$
a_{0}^{2}=\frac{P_{T}}{\int_{f_{m}}^{f_{M}} \phi(f)}
$$

In the subsequent simulation, as described in section 3, the total energy and the pulse duration of the flat power is equal to $1 \mathrm{pJ}$ and $100 \mathrm{fs}$, respectively. Meanwhile, for the Gaussian pulse-based transmission scheme, the derivative order $n$ and the standard deviation of the Gaussian pulse $\sigma$ are set to 4 and 0.15 , respectively.

\subsection{Signal-to-noise ratio}

In order to analyse the communication performance, especially the achievable communication range, SNR of the in-vivo communication channel is investigated, which can be written as a function of the transmission distance and frequency,

$$
S N R(r, f)=\frac{S(f) A_{P L}^{-1}(r, f)}{S_{N}(r, f)}
$$


where $S$ stands for the p.s.d of the transmitted signal, $A_{P L}$ denotes the channel path loss and $S_{N}$ refers to the molecular absorption noise p.s.d.

In our analysis, because the pulse energy is high enough to enable the background noise to be neglected and the self-induced noise is the dominant noise source for in-vivo nanonetworks and the self-induced noise is directly proportional to the transmitted signal, with the simplification of (18), it can be easily concluded that SNR is independent of the transmitted signal under this circumstance.

For the considered human tissue types, i.e., blood, skin and fat, the two communication schemes (flat and Gaussian-shaped pluses) result in similar SNR values as shown in Fig. 5. It allows us to conclude that the SNR can be independent of the transmitted signal power, when high pulse energy is transmitted, which enables the self-induced noise to be the dominant noise source for the in-vivo nano-networks at the $\mathrm{THz}$ band.

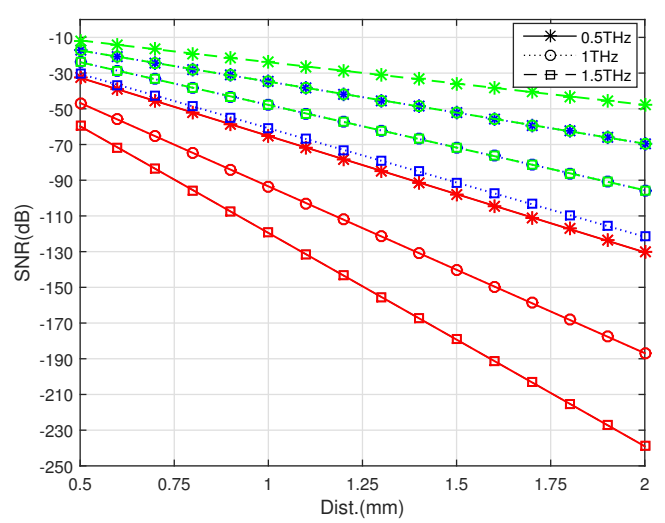

(a) Flat pulse

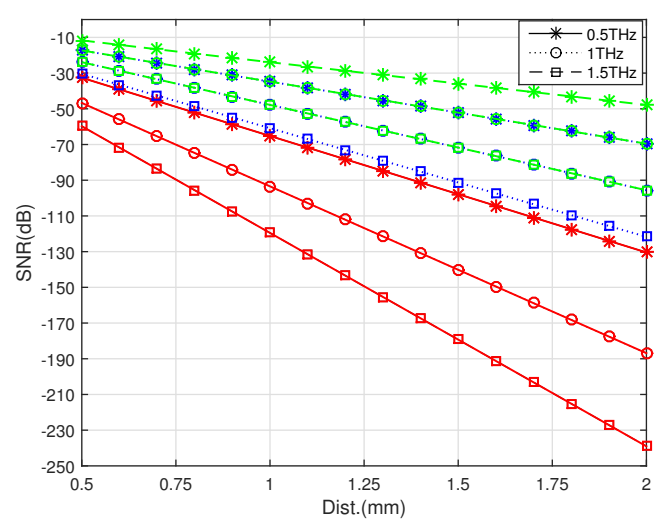

(b) Gaussian pulse

Figure 5: Channel SNR as a function of transmission distance for different power allocation schemes in human human blood (red), skin (blue) and fat (green) tissues

Furthermore, the SNR decreases with the increase of the distance and the frequency regardless of the composition of the human tissues. Apart from the dependence on the transmission distance and frequency, the water concentration in the medium also plays a significant role. Table. 1 summarises the typical $\mathrm{SNR}$ for $\mathrm{THz}$ communication in human skin, fat and blood tissues at different transmission distances for three frequencies $(0.5,1$ and $1.5 \mathrm{THz}$ ). Comparatively, the SNR in human skin is lower than the case in human fat at the same distance and frequency due to the lower water concentration in human skin tissue, and the values reduce below $-80 \mathrm{~dB}$ at $1.5 \mathrm{THz}$, when the transmission distance tends to $2 \mathrm{~mm}$ for both skin and fat. However, the SNR of human blood with the highest water concentration represents severe scenario: the value reduces much faster, which is around -80 $\mathrm{dB}$ at $1 \mathrm{THz}$ for distance of $1 \mathrm{~mm}$ but drops to $-239 \mathrm{~dB}$ at $1.5 \mathrm{THz}$, with the transmission distance going to $2 \mathrm{~mm}$. It is indicated that SNR degrades rapidly with the increase of the water concentration in the communication medium. 
Table 1: Signal-to-noise ration of human blood, skin and fat

\begin{tabular}{|c|c|c|c|c|c|c|c|c|c|}
\hline Tissues & \multicolumn{3}{|c|}{ Blood SNR (dB) } & \multicolumn{4}{c|}{ Skin SNR (dB) } & \multicolumn{5}{c|}{ Fat SNR (dB) } \\
\hline Freq. (THz) & $1 \mathrm{~mm}$ & $1.5 \mathrm{~mm}$ & $2 \mathrm{~mm}$ & $1 \mathrm{~mm}$ & $1.5 \mathrm{~mm}$ & $2 \mathrm{~mm}$ & $1 \mathrm{~mm}$ & $1.5 \mathrm{~mm}$ & $2 \mathrm{~mm}$ \\
\hline 0.5 & -65 & -98 & -130 & -35 & -52 & -70 & -24 & -36 & -48 \\
\hline 1 & -93 & -140 & -187 & -48 & -72 & -96 & -35 & -52 & -70 \\
\hline 1.5 & -120 & -180 & -239 & -61 & -91 & -122 & -48 & -72 & -96 \\
\hline
\end{tabular}

In light of the state of the art in communication devices and to an effort to make the in-vivo $\mathrm{THz}$ nano-networks realistic, it can be concluded that the maximum achievable transmission distance of in-vivo $\mathrm{THz}$ nano-networks should be restrained to approximately 1 to $2 \mathrm{~mm}$. While more specific transmission distance limitation depends on the composition of the transmission medium especially the water concentration of the medium; the operation band of in-vivo $\mathrm{THz}$ nano-networks is limited to the lower band of the $\mathrm{THz}$ band, which tends to be about $1 \mathrm{THz}$. The results further encourage the use of cooperative networking and also hybrid communication techniques using molecular and EM methods.

Although the communication distance is strongly limited in the $\mathrm{THz}$ band, this distance is estimated to be sufficient for the dense in-vivo nano-networks. For example, in Wireless Nano-sensor Networks (WNSNs), the density of nano nodes is extremely high, which is in the order of hundreds of nano-sensors per square millimetre [16], hence making the communication distance acceptable in nano-networks.

\section{Information rate}

In order to evaluate the effect of the molecular absorption noise on the performance of $\mathrm{THz}$ communication inside the human body, the communication scheme is taken into account in this section. Due to the fact that nano-communication at the $\mathrm{THz}$ band is strictly energy and distance limited, hence carrier-less communication scheme can be better adapted [30]. Based on the expected capabilities of an EM nano-transceiver, TS-OOK is proposed to be the communication scheme, where a logical " 1 " is transmitted by using a femtosecond-long pulse and a logical "0" is transmitted as silence[16].

As the molecular absorption noise is dependent on the transmitted signal, the theory using SNR to calculate channel capacity is not valid. Alternatively, the maximal mutual information rate is calculated by the transmitted and received signals to quantify the potential of $\mathrm{THz}$ band for communication inside the human body with the use of TS-OOK communication paradigm.

Utilising TS-OOK, only the background noise is present, when transmitting silence for a logical "0", and the noise power can be obtained by integration of (13) over the bandwidth as,

$$
N_{0}=\int_{B} B\left(T_{0}, f\right) \frac{c^{2}}{4 \pi\left(n_{0} f_{0}\right)^{2}} d f
$$


While both the background noise and the self-induced noise are present, when transmitting a pulse for a logical "1", and the noise power can be written as,

$$
N_{1}=\int_{B}\left(B\left(T_{0}, f\right) \frac{c^{2}}{4 \pi\left(n_{0} f_{0}\right)^{2}}+S(f)\left(1-e^{-\alpha(f) r}\left(\frac{c}{4 \pi r n f}\right)^{2}\right) d f\right.
$$

where $B$ is the channel bandwidth.

The maximum achievable information rate in bit/symbol of a communication system for a specific modulation scheme is given by the well-known Shannon Limit Theorem [31],

$$
I R=\max _{X}\{H(X)-H(X \mid Y)\}[\text { bit/symbol] }
$$

where $X$ refers to the source of information, Y stands for the output of the channel, $H(X)$ refers to the entropy of the source $X$, and $H(X \mid Y)$ stands for the conditional entropy of $X$ given $\mathrm{Y}$ or the equivocation of the channel. When using TS-OOK, the source $X$ can be modeled as a discrete binary random variable. Therefore, the entropy of the source $H(X)$ is given by [31],

$$
H(X)=-\sum_{m=0}^{1} p_{X}\left(x_{m}\right) \log _{2} p_{X}\left(x_{m}\right)
$$

where $p_{X}\left(x_{m}\right)$ refers to the probability of transmitting the symbol $m=0,1$, i.e., the probability to stay silent or to transmit a pulse, respectively.

Since the molecular absorption noise can be modelled as Additive Coloured Gaussian Noise (ACGN) [32], the probability density function (p.d.f) $N$ of the molecular absorption noise at the receiver conditioned to the transmission of symbol $x_{m}$ is given by [33],

$$
f_{N}\left(n \mid X=x_{m}\right)=\frac{1}{\sqrt{2 \pi N_{m}}} e^{-\frac{n^{2}}{2 N_{m}}}
$$

where $n$ refers to noise and $N_{m}$ refers to the molecular absorption noise power when symbol $m$ is transmitted in (19-20).

When considering a 1-bit hard receiver based on power detection, the system becomes a Binary Asymmetric Channel (BAC) and $Y$ is a discrete random variable. This channel can be fully characterised by the four transition probabilities [33],

$$
\begin{gathered}
p_{Y}(Y=0 \mid X=0)=\int_{t h 1}^{t h 2} f_{Y}(y \mid X=0) d y \\
p_{Y}(Y=1 \mid X=0)=1-p_{Y}(Y=0 \mid X=0) \\
p_{Y}(Y=0 \mid X=1)=\int_{t h 1}^{t h 2} f_{Y}(y \mid X=0) d y \\
p_{Y}(Y=1 \mid X=1)=1-p_{Y}(Y=0 \mid X=1)
\end{gathered}
$$


where $t h_{1}$ and $t h_{2}$ are two threshold values and $f_{Y}(y \mid X=x)$ is the p.d.f of the channel output $Y$ conditioned to the transmission of the symbol $X=x$, which is given by [33],

$$
f_{Y}\left(y \mid X=x_{m}\right)=\delta\left(y-a_{m}\right) * f_{N}\left(n=y \mid X=x_{m}\right)=\frac{1}{\sqrt{2 \pi N_{m}}} e^{-\frac{\left(n-a_{m}\right)^{2}}{2 N_{m}}}
$$

where $\delta$ stands for the Dirac delta function, $a_{m}$ refers to the received symbol amplitude, obtained from (5).

Contrary to the classical symmetric additive Gaussian noise channel, in the asymmetric channel, there are two points at which $f_{Y}(y \mid X=0)$ and $f_{Y}(y \mid X=1)$ intersect. It is considered these thresholds to be defined for the case without interference [33]. Therefore, $t h_{1}$ and $t h_{2}$ can be analytically computed from the intersection between two Gaussian distribution $N\left(0, N_{0}\right)$ and $N\left(0, N_{1}\right)$ respectively, which results in [33],

$$
\pm \frac{t h_{1,2}=\frac{a_{1} N_{0}}{N_{0}-N_{1}}}{N_{0}-N_{1}}
$$

The equivocation of the channel $H(X \mid Y)$ for the BAC is given by [33],

$$
\begin{array}{r}
H_{B A C}(X \mid Y)=\sum_{y=0}^{1} \sum_{x=0}^{1} p_{Y}(Y=y \mid X=x) p_{X}(X=x) \\
\log _{2}\left(\frac{\sum_{q=0}^{1} p_{Y}(Y=y \mid X=q) p_{X}(X=q)}{p_{Y}(Y=y \mid X=x) p_{X}(X=x)}\right)
\end{array}
$$

Finally, the maximum achievable information rate in bit/second is obtained by multiplying the rate in bit/symbol (21) by the rate at which symbols are transmitted, $R=1 / T_{s}=$ $1 /\left(\beta T_{p}\right)$, where $T_{s}$ is the time between symbols, $T_{p}$ is the pulse length, and $\beta$ is the ratio between them. If we assume that the $B T_{p} \simeq 1$, where $B$ stands for the channel bandwidth, the rate in bit/second is given by [33],

$$
I R_{u}=\frac{B}{\beta} I R_{u_{s y m}}
$$

If $\beta=1$, i.e., all the symbols (pulses or silences) are transmitted in a burst, and the maximum rate per nano-device is achieved, provided that the incoming information rate and the read-out rate to and from the nano-transceiver can match the channel rate. By increasing $\beta$, the single-user rate is reduced, but the requirements on the transceiver are greatly relaxed.

In this paper, the bandwidth of the $\mathrm{THz}$ communication channel is considered to be 1 $\mathrm{THz}$, and the information rate for three considered human tissues has been studied and the results are shown in Fig. 6. It can be clearly seen that the information rate tends to 
be 1 Terabits per second (Tbps) for communication in three kinds of human tissue, when the communication distance is $0.5 \mathrm{~mm}$. The information rate decreases steadily with the transmission distance regardless of the medium type, and reaches about 0.96 Tbps when the transmission distance is further increased to $2 \mathrm{~mm}$. Differently, the information rate presents the best case in human fat, because it has the least water concentration comparing with human blood and skin. The main reason for such high information rate is the extremely high bandwidth for the $\mathrm{THz}$ communication. The obtained information rate indicates that complex tasks can be completed in the envisioned nano-communication inside the human body by the high ability of successfully transmitting information over the communication channel.

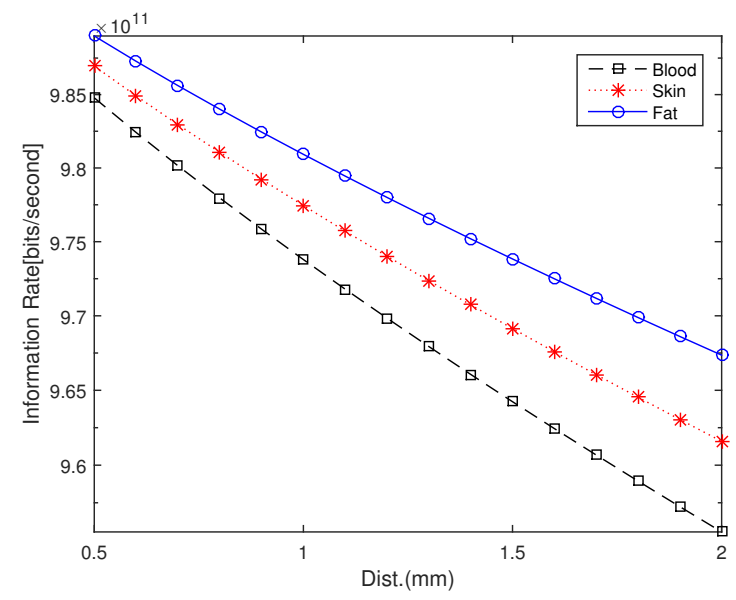

Figure 6: Information rate as a function of transmission distance for different human tissues

\section{Conclusion}

In this paper, the molecular absorption noise model for the in-vivo nano-communication links and investigation on the physical principles and mathematical derivations of the background and self-induced noise are presented. The channel noise is dominantly contributed by the self-induced noise and is dependent on the transmitted signal power allocation schemes, which motivates the investigation of SNR on different transmitted power schemes. The study highlights that the SNR is independent of the power allocation schemes mainly because of the signal-dependent molecular absorption. Moreover, the results indicate that the maximum achievable transmission distance of in-vivo $\mathrm{THz}$ nano-networks should be restrained to approximately 1 to $2 \mathrm{~mm}$. While more specific transmission distance limitation depends on the composition of the transmission medium, especially the water concentration of the medium; and the operation band of in-vivo THz nano-networks is limited to the lower band of the $\mathrm{THz}$ band, which tends to be about $1 \mathrm{THz}$. The information rate decreases steadily with the transmission distance regardless of the medium type, and reaches about $0.96 \mathrm{Tbps}$ when the transmission distance is further increased to $2 \mathrm{~mm}$. These results motivate further analysis in hierarchical/cooperative networking and the development of hybrid communication techniques i.e., a combination of molecular and EM methods. 


\section{Acknowledgement}

Many thanks to the CSC (China Scholarship Council) for supporting the first authors research studies at Queen Mary University of London (QMUL), UK. This publication was made possible by NPRP grant \# 7-125-2-061 from the Qatar National Research Fund (a member of Qatar Foundation). The statements made herein are solely the responsibility of the authors.

\section{References}

[1] I. F. Akyildiz, F. Brunetti, C. Blzquez, Nanonetworks: A new communication paradigm, Computer Networks 52 (12) (2008) 2260-2279. doi:10.1016/j.comnet.2008.04.001.

[2] I. F. Akyildiz, J. M. Jornet, Electromagnetic wireless nanosensor networks, Nano Communication Networks 1 (1) (2010) 3-19. doi:10.1016/j.nancom.2010.04.001.

[3] I. F. Akyildiz, J. M. Jornet, The internet of nano-things, Wireless Communications, IEEE 17 (6) (2010) $58-63$.

[4] H. Guo, P. Johari, J. M. Jornet, Z. Sun, Intra-body optical channel modeling for in vivo wireless nanosensor networks, IEEE transactions on nanobioscience 15 (1) (2016) 41-52.

[5] G. Piro, K. Yang, G. Boggia, N. Chopra, L. A. Grieco, A. Alomainy, Terahertz communications in human tissues at the nanoscale for healthcare applications, IEEE Transactions on Nanotechnology 14 (3) (2015) 404-406.

[6] Q. H. Abbasi, H. El Sallabi, N. Chopra, K. Yang, K. A. Qaraqe, A. Alomainy, Terahertz channel characterization inside the human skin for nano-scale body-centric networks, IEEE Transactions on Terahertz Science and Technology 6 (3) (2016) 427-434.

[7] A. Fitzgerald, E. Berry, N. Zinov'ev, S. Homer-Vanniasinkam, R. Miles, J. Chamberlain, M. Smith, Catalogue of human tissue optical properties at terahertz frequencies, Journal of Biological Physics $29(2-3)(2003)$ 123-128.

[8] E. Berry, A. J. Fitzgerald, N. N. Zinov'ev, G. C. Walker, S. Homer-Vanniasinkam, C. D. Sudworth, R. E. Miles, J. M. Chamberlain, M. A. Smith, Optical properties of tissue measured using terahertzpulsed imaging, in: Medical Imaging 2003, International Society for Optics and Photonics, 2003, pp. $459-470$.

[9] J. M. Jornet, I. F. Akyildiz, Channel capacity of electromagnetic nanonetworks in the terahertz band, in: Communications (ICC), 2010 IEEE International Conference on, IEEE, 2010, pp. 1-6, the noise temperature and power are calculated in this paper.

[10] J. M. Jornet, I. F. Akyildiz, Channel modeling and capacity analysis for electromagnetic wireless nanonetworks in the terahertz band, Wireless Communications, IEEE Transactions on 10 (10) (2011) 3211-3221.

[11] K. Yang, A. Pellegrini, M. O. Munoz, A. Brizzi, A. Alomainy, Y. Hao, Numerical analysis and characterization of thz propagation channel for body-centric nano-communications, IEEE TRANSACTIONS ON TERAHERTZ SCIENCE AND TECHNOLOGY, VOL. 5, NO. 3.

[12] P. Boronin, V. Petrov, D. Moltchanov, Y. Koucheryavy, J. M. Jornet, Capacity and throughput analysis of nanoscale machine communication through transparency windows in the terahertz band, Nano Communication Networks 5 (3) (2014) 72-82.

[13] P. Boronin, D. Moltchanov, Y. Koucheryavy, A molecular noise model for thz channels, in: Communications (ICC), 2015 IEEE International Conference on, IEEE, 2015, pp. 1286-1291.

[14] Q. H. Abbasi, A. Alomainy, M. U. Rehman, K. Qaraqe, Advances in Body-Centric Wireless Communication: Applications and State-of-the-Art, The Institution of Engineering and Technolog (IET) Publication, 2016.

[15] P. Wang, J. M. Jornet, M. A. Malik, N. Akkari, I. F. Akyildiz, Energy and spectrum-aware mac protocol for perpetual wireless nanosensor networks in the terahertz band, Ad Hoc Networks 11 (8) (2013) 2541-2555. 
[16] J. M. Jornet, I. F. Akyildiz, Femtosecond-long pulse-based modulation for terahertz band communication in nanonetworks, Communications, IEEE Transactions on 62 (5) (2014) 1742-1754.

[17] M. Pierobon, J. M. Jornet, N. Akkari, S. Almasri, I. F. Akyildiz, A routing framework for energy harvesting wireless nanosensor networks in the terahertz band, Wireless networks 20 (5) (2014) 11691183.

[18] N. Akkari, J. Jornet, P. Wang, E. Fadel, L. Elrefaei, M. Malik, S. Almasri, I. Akyildiz, Joint physical and link layer error control analysis for nanonetworks in the terahertz band, Wireless Networks (2015) $1-13$.

[19] J. Kokkoniemi, J. Lehtomäki, M. Juntti, A discussion on molecular absorption noise in the terahertz band, Nano Communication Networks 8 (2016) 35-45.

[20] R. Zhang, K. Yang, A. Alomainy, Q. H. Abbasi, K. Qaraqe, R. M. Shubair, Modelling of the terahertz communication channel for in-vivo nano-networks in the presence of noise, in: Microwave Symposium (MMS), 2016 16th Mediterranean, IEEE, 2016, pp. 1-4.

[21] S. Paine, The am atmospheric model (sma tech. memo 152; cambridge: Harvard univ.) (2004).

[22] M. Fox, Optical properties of solids. oxford master series in condensed matter physics (2001).

[23] J. Kokkoniemi, J. Lehtomäki, K. Umebayashi, M. Juntti, Frequency and time domain channel models for nanonetworks in terahertz band, IEEE Transactions on Antennas and Propagation 63 (2) (2015) 678-691.

[24] S. Chandrasekhar, Radiative transfer, Courier Corporation, 2013.

[25] A. K. Geim, K. S. Novoselov, The rise of graphene, Nature materials 6 (3) (2007) 183-191.

[26] A. N. Pal, A. Ghosh, Ultralow noise field-effect transistor from multilayer graphene, Applied Physics Letters 95 (8) (2009) 082105.

[27] J. M. Jornet, I. F. Akyildiz, Graphene-based plasmonic nano-transceiver for terahertz band communication, in: The 8th European Conference on Antennas and Propagation (EuCAP 2014), IEEE, 2014, pp. $492-496$.

[28] V. Ryzhii, M. Ryzhii, V. Mitin, T. Otsuji, Toward the creation of terahertz graphene injection laser, Journal of Applied Physics 110 (9) (2011) 094503.

[29] A. Goldsmith, Wireless communications, Cambridge university press, 2005.

[30] E. Zarepour, M. Hassan, C. T. Chou, S. Bayat, Performance analysis of carrier-less modulation schemes for wireless nanosensor networks, in: Nanotechnology (IEEE-NANO), 2015 IEEE 15th International Conference on, IEEE, 2015, pp. 45-50.

[31] C. E. Shannon, A mathematical theory of communication, ACM SIGMOBILE Mobile Computing and Communications Review 5 (1) (2001) 3-55.

[32] J. M. Jornet, I. F. Akyildiz, Information capacity of pulse-based wireless nanosensor networks, in: Sensor, Mesh and Ad Hoc Communications and Networks (SECON), 2011 8th Annual IEEE Communications Society Conference on, IEEE, 2011, pp. 80-88.

[33] J. M. Jornet, Low-weight error-prevention codes for electromagnetic nanonetworks in the terahertz band, Nano Communication Networks 5 (1) (2014) 35-44. 